\title{
EDITORIAL
}

\section{THE COMPARISON OF SERUM TESTS FOR SYPHILIS}

While discussing cardiolipin antigens in an address delivered a few years ago, Dr. Earle Moore referred to serologists as "individualistic prima donnas", because they had tried to adapt cardiolipin antigens to their own techniques but had failed to reach any agreement upon the most suitable test. There has been a progressive increase in the sensitivity of tests and it is not sufficiently appreciated that this may sometimes only be achieved at the expense of specificity. In some of the supersensitive screening procedures, specificity has been deliberately sacrificed in order to save time and labour in the examination of large numbers of sera. At the same time, serum tests for syphilis are being increasingly applied to healthy people, as, for example, pregnant women and blood donors, and in the United States mass surveys of civilians have been carried out and members of the Services have been examined on demobilization. Since many non-specific reactions were found in this last group, interest has been aroused in these so-called biological false positive reactions, and it is coming to be recognized that these present a problem of considerable magnitude. Thus a satisfactory test may have a specificity of 99.8 per cent., but if a laboratory uses it to examine 500 sera a week, about fifty non-specific reactions a year may be expected.

In Great Britain very little information is available about the specificity and sensitivity levels of reactions used in the diagnosis of syphilis. Tests are often described very loosely; the term "Wassermann reaction", for example, covers a multitude of methods and modifications, and it is often difficult for clinicians to interpret the results of tests carried out on patients' sera in different laboratories. Attempts were made to measure the performance of standard tests carried out by author serologists at the International Serological Conferences held under the auspices of the League of Nations, and this programme is to be continued by the World Health Organization. In the United States parallel serum testing is regularly carried out in the State laboratories, and publication of the results makes information about the specificity and sensitivity rating of the tests in current use easily available to clinicians.

One of the main difficulties encountered in arranging the parallel testing of sera in more than a few laboratories is the collection of enough material from patients whose clinical status is known. Thus, if twenty laboratories participate, a minimum of $50 \mathrm{ml}$. serum is needed from each patient. The use of freeze-drying methods obviates many of the difficulties of storage and deterioration of sera before testing. If small numbers of test sera are distributed at weekly or fortnightly intervals, they can be included in the routine tests of the participating laboratories, thus giving very little extra work. The frequent examination of a few sera, rather than the annual testing of a large number, allows the early detection of any divergence from the usual performance of the routine tests.

In Great Britain little has been done to explore these possibilities. Judging from American experience, advantages might accrue both to pathologists, who would be able to compare the performance of their techniques with those of their colleagues, and to clinicians, who would gain additional information about the merits and limitations of the tests carried out in the laboratories serving their clinics. The scheme would encourage cooperation between clinician and pathologist, and on this ground alone merits consideration. 